\title{
Evolution and Diversity of Assembly-Line Polyketide Synthases
}

Aleksandra Nivinaa,b, Kai P. Yuet ${ }^{a, b}, J^{a k e} H_{s u}^{b, c}$, and Chaitan Khosla ${ }^{a, b, c,}{ }^{*}$

aDepartment of Chemistry, bStanford ChEM-H, CDepartment of Chemical Engineering Stanford University, Stanford, CA 94305, USA

*Corresponding author, khosla@stanford.edu

\section{Supplementary information}

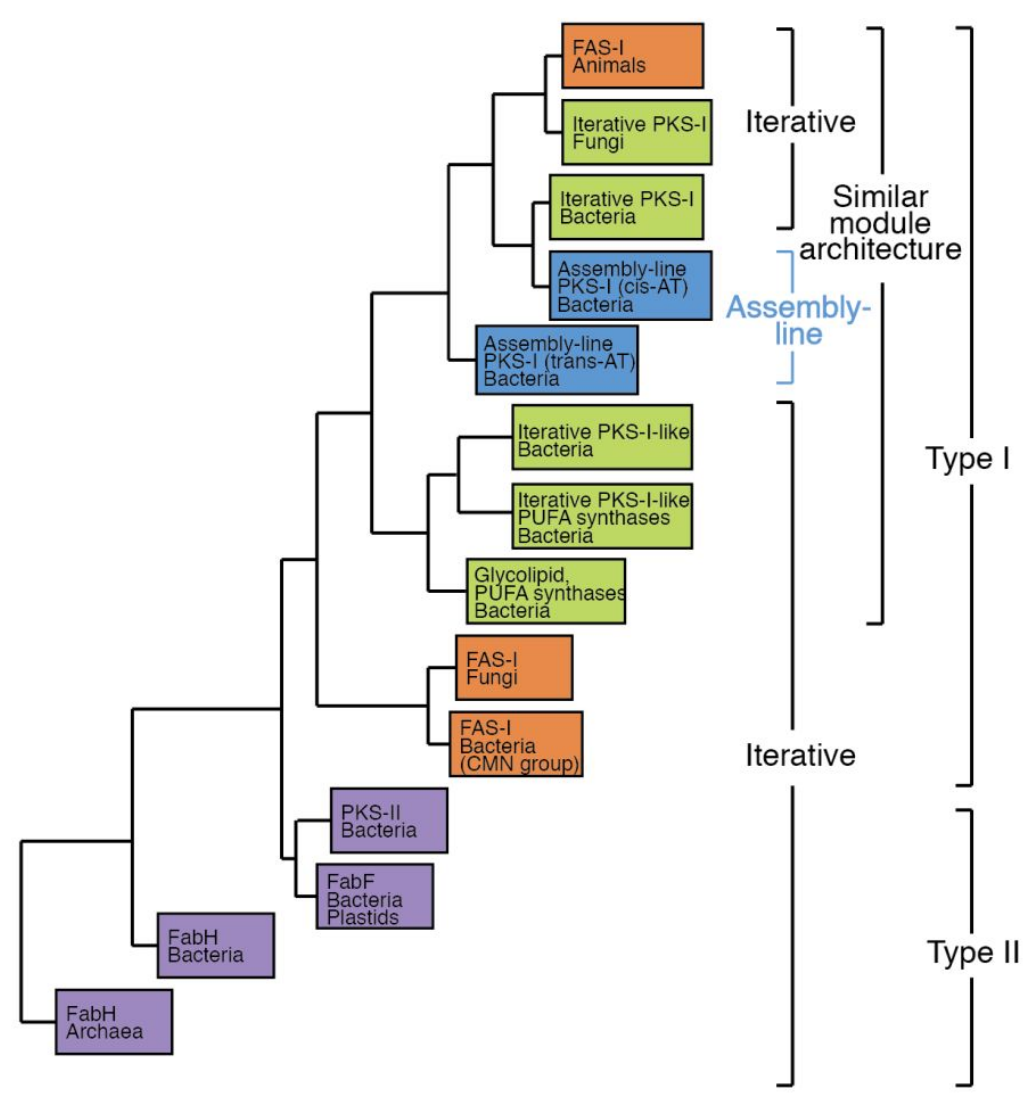

Supplementary Figure S1. Schematic representation of phylogenetic relationships between KS domains from different types of FASs and PKSs, as inferred by Bayesian statistics. Adapted from Jenke-Kodama et al. $2005^{1}$ and Jenke-Kodama et al. 2009. ${ }^{2}$ 




Supplementary Figure S2. Network of 3,551 distinct assembly-line PKS clusters, visualized by Cytoscape $3.7 .2^{3}$. Nodes correspond to known clusters (larger circles) and "orphans" (smaller circles), color coded according to the phylum of their host (graphical legend). Edges represent $>50 \%$ sequence similarity between two clusters, calculated as described in ${ }^{4}$.

The catalog, the dendrogram and the network file are available online: http://web.stanford.edu/group/orphan_pks/

The code used in this work is available on GitHub: https://github.com/aleksnivina/Orphan_PKS_catalog

(1) Jenke-Kodama, H.; Sandmann, A.; Müller, R.; Dittmann, E. Evolutionary Implications of Bacterial Polyketide Synthases. Mol. Biol. Evol. 2005, 22, 20272039.

(2) Jenke-Kodama, H.; Dittmann, E. Evolution of Metabolic Diversity: Insights From Microbial Polyketide Synthases. Phytochemistry 2009, 70, 1858-1866.

(3) Shannon, P.; Markiel, A.; Ozier, O.; Baliga, N. S.; Wang, J. T.; Ramage, D.; Amin, N.; Schwikowski, B.; Ideker, T. Cytoscape: a Software Environment for Integrated Models of Biomolecular Interaction Networks. Genome Res. 2003, 13, 2498-2504.

(4) O'Brien, R. V.; Davis, R. W.; Khosla, C.; Hillenmeyer, M. E. Computational Identification and Analysis of Orphan Assembly-Line Polyketide Synthases. J. Antibiot. 2014, 67, 89-97. 